www.jmscr.igmpublication.org

Index Copernicus Value: 79.54

ISSN (e)-2347-176x ISSN (p) 2455-0450

crossrefDOI: https://dx.doi.org/10.18535/jmscr/v7i3.73

\title{
Clinical Profile of Metastatic Cancer to the Brain in a Tertiary Care Hospital
}

\author{
Authors \\ Kannan Jayaraman*, Ramya Rangarajan \\ *Corresponding Author \\ Kannan Jayaraman
}

\section{Introduction}

Metastatic cancer to the brain is associated with a very grave prognosis. Secondaries brain is the most common adult intracranial neoplasm ${ }^{(1)}$. Around $10 \%$ of cancer patients can develop brain metastases at some point during their lifetime ${ }^{(2,3)}$. The incidence of brain metastases seems to be on the rise due to better treatment of primary disease, longer survival of cancer patients due to effective treatment modalities and advanced imaging technologies.

Certain primary cancers like lung, breast, renal cell cancer and melanoma have an increased propensity to metastasise to the brain. Lung cancer is the most common malignancy to metastasise to the brain accounting for $40-50 \%^{(4)}$ of brain metastases followed by breast cancer (15-25\%), melanoma (5-25\%) and renal cell cancer (5-10\%). Synchronous metastases in other sites are commonly seen with brain metastases. Isolated brain metastases in the absence of other systemic sites are rare. Isolated metastasis in the brain without a detectable primary cancer is seen in about $5-10 \%{ }^{(5)}$.

The most common site of metastases is in the supratentorial region. $80 \%$ of metastases occur in the cerebral hemispheres ${ }^{(4,6)}$. Cerebellum and brainstem account for $15 \%$ and $5 \%$ of metastatic sites. Patients commonly present with headache, vomiting, blurring of vision, seizures and neurologic deficits. The median survival of patients with brain metastases without treatment is 1 month.

In this study, we analysed the clinical profile of brain metastases patients who presented to department of oncology from May 2013 to May 2015.

\section{Materials and Methods}

We analysed the case records of brain metastases patients who attended the department of oncology between May 2013 and May 2015. Age, gender, clinical presentation, primary site, histopathology of primary, median duration from diagnosis of cancer to brain metastases, site of metastases, treatment given were recorded.

\section{Results}

There were 35 patients of brain metastases between May 2013 and May 2015. In the present study, majority of patients were females $(77.14 \%)$. (Fig1) The mean age of patients presenting with 


\section{JMSCR Vol||07||Issue ||03||Page 400-404||March}

brain metastases was 47 years. Majority of patients were between the fourth and fifth decade. (Fig 2). Breast cancer was the most common primary metastasizing to the brain followed by lung, melanoma, unknown primary, ovary, tongue, gastrointestinal stromal tumor and thyroid (Fig 3). $40 \%$ of patients had synchronus bone metastases along with brain metastases. $31.43 \%$ had lung metastases along with brain metastases. Brain was the only site of metastases in $11.43 \%$ of patients. Multiple site of metastases were found in $17.14 \% \%$ of patients. (Fig 4). $40 \%$ of our patients presented with brain metastases at the time of diagnosis. The time from diagnosis to development of brain metastases was less than 5 years in $42.86 \%$ of patients. $17.14 \%$ of patients presented with brain metastases after 5 years of initial diagnosis. (Fig 5).

The most common location of brain metastases was in the supratentorial region $(88.57 \%) .8 .57 \%$ of patients had metastases both in the supratentorial and infratentorial locations. Only $2.86 \%$ of our patients had metastases in the infratentorial location. (Fig 6). 54.29\% of patients had multiple sites of metastases whereas $45.71 \%$ of patients had solitary brain metastases. (Fig 7). All our patients were treated with whole brain radiotherapy. The palliative dose fractionation schedule was 30Gy in 10 fractions.

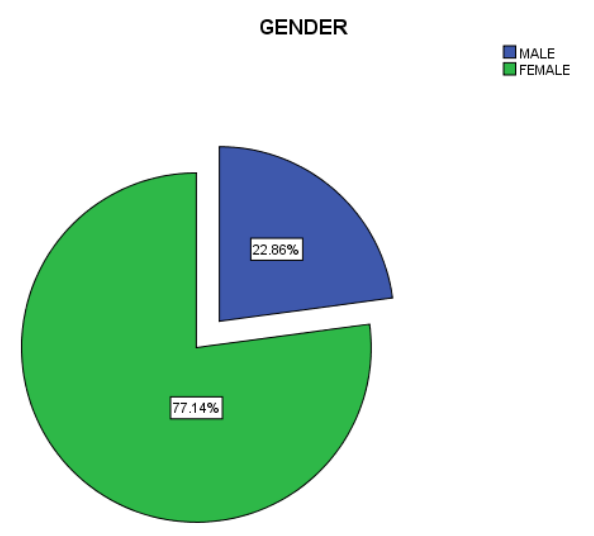

Fig 1: Pie diagram showing gender distribution

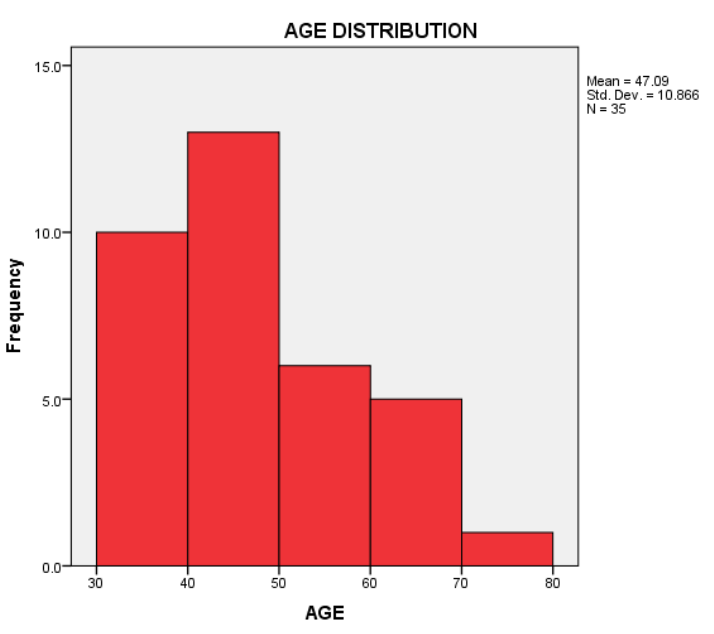

Fig 2: Bar diagram showing age distribution

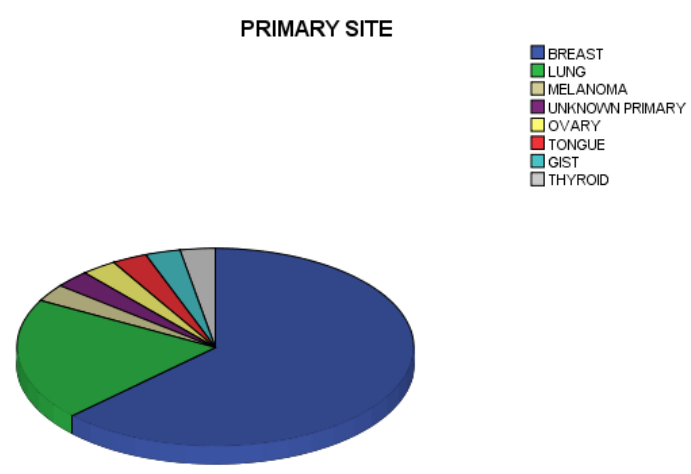

Fig 3: Pie diagram showing the primary site

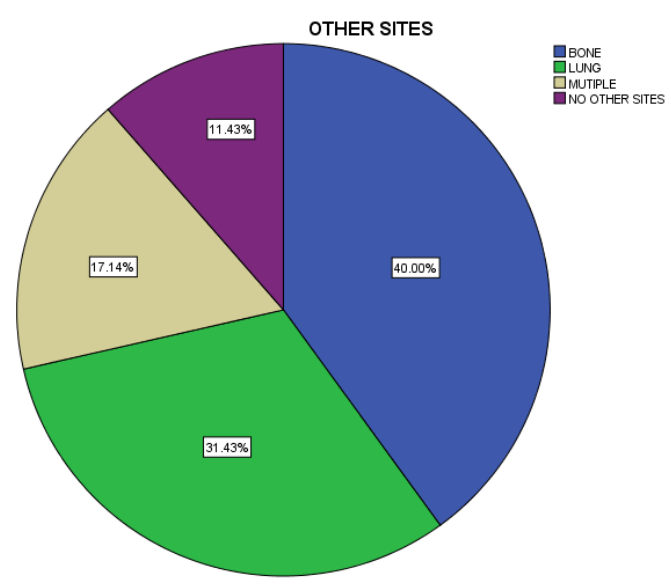

Fig 4: Pie diagram showing other sites of metastases 


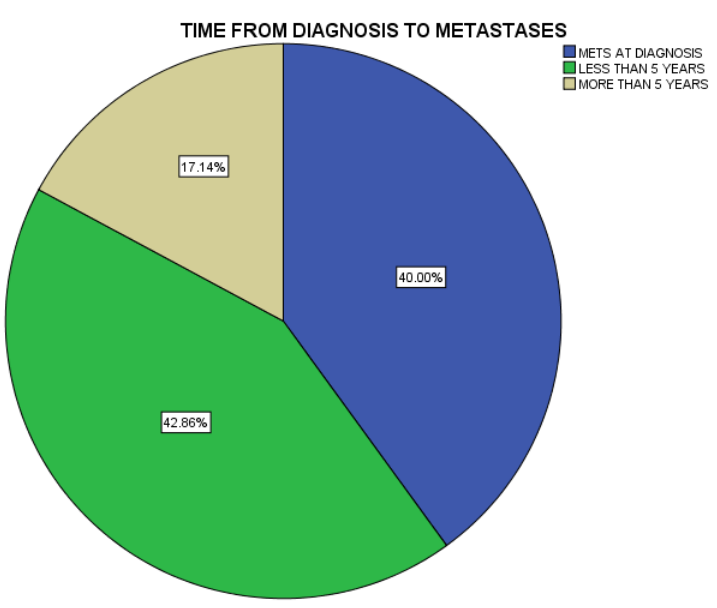

Fig 5: Time from diagnosis to development of brain metastases

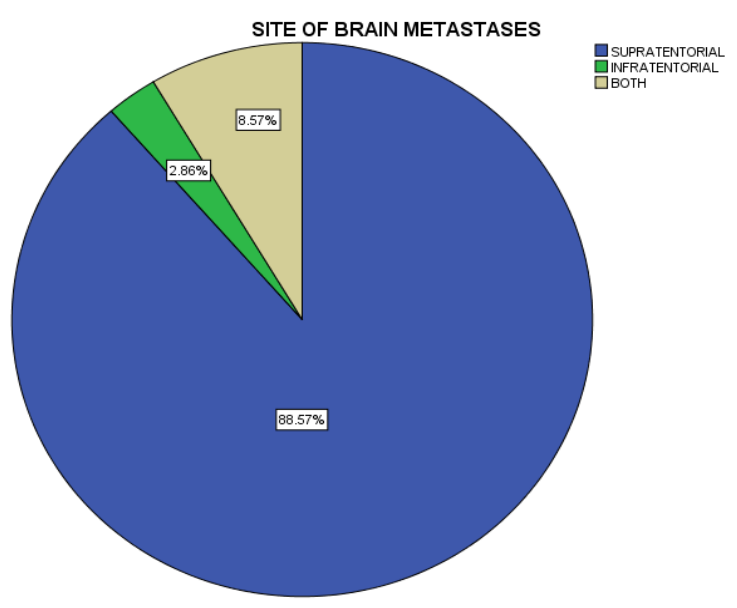

Fig 6 : Location of brain metastases

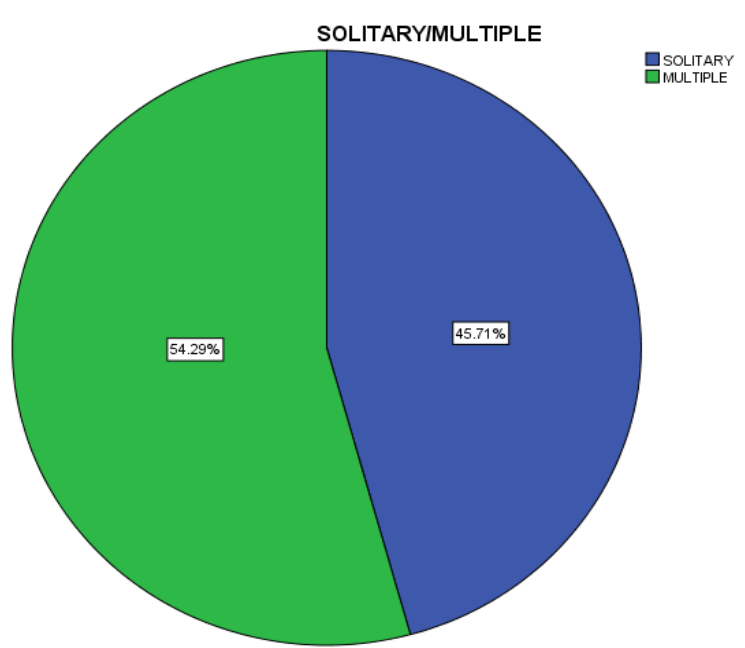

Fig 7: Solitary versus multiple metastases

\section{Discussion}

The variables affecting prognosis in a patient with brain metastases include age, performance status, presence of single or multiple metastases, the primary tumor type and the extent of systemic disease, duration from development of primary to development of brain metastases ${ }^{(7-11)}$. Patients commonly present with headache or seizures. Meningeal involvement can lead to nuchal rigidity and photophobia. The timing of onset of symptoms is usually sub acute. Vascular etiology like bleeding can lead to acute onset of symptoms. Cognitive dysfunction and motor dysfunction can also occur in a third of patients. Headache was the most common presenting symptom in all our patients. One fourth of our patients had associated vomiting along with headache.

Brain metastases occur most commonly in patients aged between 50 and 70 years ${ }^{(12,13)}$. Some studies have reported a slightly higher incidence of brain metastases in females compared to males ${ }^{(14)}$ whereas a few other studies have reported no significant gender based differences in the incidence of brain metastases ${ }^{(15)}$. Our study showed a female preponderance. The most common histologic subtype metastasizing to the brain is adenocarcinoma, followed by small cell lung carcinoma and squamous cell carcinoma (13, 16, 17). Primary tumor histology remains unknown in 3 to $4 \%$ of patients ${ }^{(18)}$. The most common primary metastasizing to the brain is the lung cancer, followed by breast cancer, colorectal cancer, melanoma and unknown primary ${ }^{(19)}$. Breast cancer was the most common primary to metastasize to the brain in our study.

The most common location of the metastases is the supratentorial region ${ }^{(18)}$ namely the cerebrum followed by cerebellum and brainstem. The lower flow posterior circulation explains the lower incidence of metastases in the cerebellum and brainstem compared to the cerebrum. Mutiple metastases in the brain are found to be more common than single metastasis ${ }^{(18)}$. The location of brain metastasis determines the clinical presentation, with headache being the most common symptom. Patients also present with vomiting, neurological deficit and seizures. Nearly two thirds of patients have an uncontrolled primary disease at the time of detection of brain metastases $^{(15)}$. 
Contrast enhanced computed tomography and contrast enhanced magnetic resonance imaging are the commonly used diagnostic modalities. Recursive partitioning analysis tool published by Gaspar et al is the most commonly used prognostic tool to estimate the length of survival for patients with brain metastases ${ }^{(20)}$. Performance status, age and the status of the primary tumor are the factors which are used to determine the prognosis of patients.

Standard treatment options for brain metastases include surgical resection, whole brain radiotherapy, stereotactic radiosurgery, systemic therapy and targeted therapy. The choice of treatment modality is determined by the size of the lesion, location of the lesion, number of metastases, histology and performance status. Surgical resection is usually advocated in a patient with a solitary 3 to $4 \mathrm{~cm}$ lesion. Whole brain radiotherapy is the standard treatment option for most patients with brain metastases. Typical dose fractionation schedule includes 30Gy in 10 fractions. A precise form of radiotherapy to target solitary lesions in the brain is stereotactic radiosurgery. Radiosurgery is used either as a single modality or in combination with whole brain radiotherapy and surgery depending on the size of the lesion. Disruption of blood brain barrier occurs in patients with brain metastases. Systemic chemotherapy and targeted therapy can attain significant concentrations in the brain because of this disruption.

\section{Conclusion}

Our study concludes that breast cancer was the most common primary to metastasize to the brain. Majority of our patients had multiple sites of metastases along with brain metastases. Less than one fourth of our patients developed brain metastases more than 5 years from the time of diagnosis. Our study adds to the existing data in the pool of literature. These data will aid in assessing the prognosis and in choosing the treatment options.

\section{References}

1. Sawaya R, Ligon BL, Bindal RK. Management of metastatic brain tumors. Ann Surg Oncol. 1994;1:169-78

2. Barnholtz-Sloan JS, Sloan AE, Davis FG, et al. Incidence proportions of brain metastases in patients diagnosed (1973 to 2001) in the Metropolitan Detroit Cancer Surveillance System. J Clin Oncol 2004;22:2865-2872.

3. Schouten LJ, Rutten J, Huveneers HA, et al. Incidence of brain metastases in a cohort of patients with carcinoma of the breast, colon, kidney, and lung and melanoma. Cancer 2002;94:2698-2705.

4. Delattre JY, Krol G, Thaler HT, Posner JB. Distribution of brain metastases. Arch Neurol. 1988;45:741-4.

5. Lagerwaard FJ, Levendag PC, Nowak PJ, Eijkenboom WM, Hanssens PE, Schmitz PI. Identification of prognostic factors in patients with brain metastases: A review of 1292 patients. Int J Radiat Oncol Biol Phys. 1999;43:795-803.

6. Ewing J Ewing J. Metastasis. In: Ewing J, editor. Neoplastic Diseases: A Treastise on Tumours. Philadelphia: Saunders; 1940. p. 62-74.

7. Gaspar L, Scott C, Rotman M, et al. Recursive partitioning analysis (RPA) of prognostic factors in three Radiation Therapy Oncology Group (RTOG) brain metastases trials. Int $J$ Radiat Oncol Biol Phys 1997;37:745-751.

8. Lagerwaard FJ, Levendag PC, Nowak PJ, et al. Identification of prognostic factors in patients with brain metastases: A review of 1292 patients. Int J Radiat Oncol Biol Phys 1999;43:795-803.

9. Yates JW, Chalmer B, McKegney FP. Evaluation of patients with advanced cancer using the Karnofsky performance status. Cancer 1980;45:2220-2224.

10. Fife KM, Colman MH, Stevens GN, et al. Determinants of outcome in melanoma 
patients with cerebral metastases. J Clin Oncol 2004;22:1293-1300.

11. Agboola O, Benoit B, Cross P, et al. Prognostic factors derived from recursive partition analysis (RPA) of Radiation Therapy Oncology Group (RTOG) brain metastases trials applied to surgically resected and irradiated brain metastatic cases. Int J Radiat Oncol Biol Phys 1998;42:155-159.

12. Fabi A, Felici A, Metro G, et al (2011). Brain metastases from solid tumors: disease outcome according to type of treatment and therapeutic resources of the treating center. J Exp Clin Cancer Res, 30, 10.

13. Saha A, Ghosh SK, Roy C, et al (2013). Demographic and clinical profile of patients with brain metastases: A retrospective study. Asian J Neurosurg, 8, 157-61.

14. Ali Akhavan, Fariba Binesh, Samaneh Heidari. Survival of Brain Metastatic Patients in Yazd, Iran Asian Pacific Journal of Cancer Prevention. 2014;15:3571-3574.

15. Victor TS. Brain metastasis. Medscape reference. [Last updated on 2011 Oct 7]. Available from:http://www. emedicine.medscape.com/article

16. Debnath H, Barua KK, Hossain MA, Khair MA, Islam MA. Outcome and prognosis of metastatic brain tumour: A study of 35 cases. Bangladesh J Neurosci. 2008;24:1723

17. Jin J, Zhou X, Liang X, et al (2011). A study of patients with brain metastases as the initial manifestation of their systemic cancer in a Chinese population. J Neurooncol, 103, 649-55.

18. Delattre JY, Krol G, Thaler HT, et al (1988). Distribution of brain metastases. Arch Neurol, 45, 741 -4.
19. Lassman AB, DeAngelis LM. Brain metastases. Neurol Clin. 2003;21:1-23.

20. Recursive partitioning analysis (RPA) of prognostic factors in three Radiation Therapy Oncology Group (RTOG) brain metastases trials. Gaspar L, Scott C, Rotman M, Asbell S, Phillips T, Wasserman T, McKenna WG, Byhardt RInt J Radiat Oncol Biol Phys. 1997 Mar 1; 37(4):745-51. 